\title{
Anisotropy of Exoemission Properties of Quartz Single Crystals
}

To cite this article: A. F. Zatsepin et al 1985 Jpn. J. Appl. Phys. 2488

\section{Related content}

- Exoelectron Emission of $\mathrm{Na}_{2} \mathrm{CO}_{3}$ in
Vacuum
Boncho Bonev
- The Progress of Research on Exoelectron
$\frac{\text { Emission and Applications in Japan }}{\text { Masaharu Kawanishi }}$
- The Adsorption of Gases on Clean Metal
Surfaces and Exoelectron Emission
J.A. Ramsey

View the article online for updates and enhancements.

\section{Recent citations}

- Monte-Carlo simulation of electron
$\frac{\underline{\text { transport in radiation-charged crystalline }}}{\text { dielectrics }}$
V. S. Kortov and S. V. Zvonarev
- Exoelectronic spectroscopy of intrinsic and
$\frac{\text { extrinsic color centers in surface lavers of }}{\text { alkali silicate glasses }}$
V.I. Arbusov et al
- Imaging and Electron Energy Analysis of
Exo-Electron Emission
I. R. Plummer and D. W. Turner




\title{
Anisotropy of Exoemission Properties of Quartz Single Crystals
}

\author{
A. F. Zatsepin, V. A. Kalentyev and V. S. Kortov
}

Ural Polytechinical Institute, Sverdlovsk, 620002, USSR

\begin{abstract}
The symmetrical aspects of exoelectron emission in quantz are discussed. The dependence of the emission intensity and the energy spectra on the emitting direction show that the anisotropy of the emission properties of quartz is determined by the point-symmetry group of a crystal. It is also shown that the absence of a symmetry center in a quartz lattice changes the charge distribution of the emission-active layer upon heating. TSEE related to piezoelectric polarization along certain crystallographic directions was obtained.
\end{abstract}

\section{§1. Introduction}

It is of primary importance that the physics of the exoemission phenomenon be clarified regarding the relations between surface characteristics and lattice geometry of a crystal. This problem is due to an observed nonequivalence of exoemission parameters regarding the real surfaces of a single crystal.

The influence of orientation upon thermally stimulated exoemission (TSEE) has been studied for LiF, $\mathrm{Al}_{2} \mathrm{O}_{3}, \mathrm{SiO}_{2}, \mathrm{Be}_{2} \mathrm{SiO}_{4}$ and $\mathrm{NaCl}$ crystals. ${ }^{1-8)}$ However, at present there has been no definite interpretation of these effects. A lack of detailed investigations has led to considerable difficulties in an analysis and generalization of the results obtained from a study of the TSEE regularities of anisotropic dielectrics. ${ }^{8}$

The object of this paper is to determine the relations between TSEE parameters and the symmetrical characteristics of a quartz lattice, as well as to reveal the possible influence of geometrical factors on the emission mechanism.

\section{§2. Experimental}

Exoemission measurements were performed in a vacuum $\left(10^{-4}-10^{-7} \mathrm{~Pa}\right)$ using a secondary-electron multiplier (VEU-6) as an electron detector. TSEE registration was made after a preliminary surface cleaning or after an exposure to air. The energy distribution of exoelectrons (EDE) was registered by the scanning retarding-potential method using a multichannel amplitude analyser.

Samples of $0.5 \times 10 \times 10 \mathrm{~mm}^{3}$ were cut from the $\langle c\rangle \mathrm{sec}-$ tor of a synthetic quartz crystal. Samples of quartz glass $(\mathrm{KV}-1)$ were studied for a comparison. The orientation of the crystal surface was fixed by the cutting angle relative to the $X, Y, Z$-axes and was determined by $\mathrm{X}$-ray diffraction. The orientation error after a mechanical treatment and etching did not exceed $\pm 15^{\prime}$. Before TSEE measurements, the samples were annealed and excited by X-rays $(V=50 \mathrm{keV}, I=15 \mathrm{~mA})$ or electron bombardment $(E=1-1,5 \mathrm{keV} ; I=10-15 \mu \mathrm{A})$. Secondaryelectron current was measured during electron excitation.

\section{§3. Results}

\section{Exoemission activity.}

Measurements showed that for identical excitation conditions the TSEE current density of crystalline samples

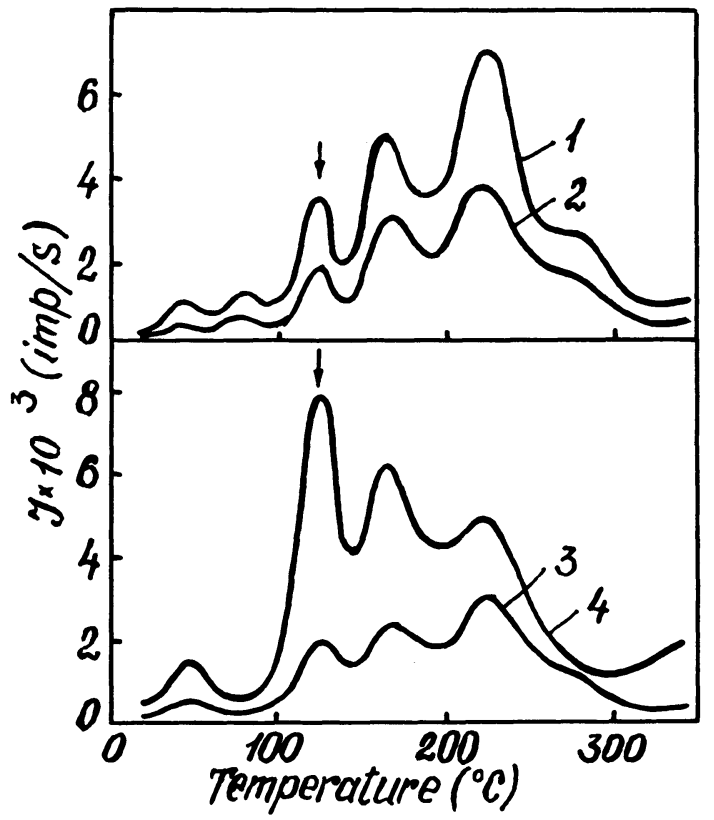

Fig. 1. TSEE from surfaces of quartz with different cuts after electron bombardment $(1 \mathrm{keV}, 10 \mu \mathrm{A})$ in a vacuum of $10^{-4} \mathrm{~Pa}: 1-X^{+}$; $2-X^{-} ; 3-Y ; 4-Z$. The arrows show the TSEE adsorption peak.

depends on orientation. Glass samples were characterized by a uniform emission activity. Figure 1 illustrates typical TSEE curves for quartz with the main crystallographic cuts after electron bombardment.

It was determined by special experiments that the registered TSEE maxima were caused by structural defect centers localized in the emission-active subsurface layer of the crystal. The TSEE peak at $120^{\circ} \mathrm{C}$ was due to absorption. It disappeared in an ultrahigh vacuum after surface cleaning and reappeared after an exposure to air.

The dependence of the TSEE peaks on crystal orientation was observed. An $X^{+}$-cut surface was characterized by the greatest emission activity in the "structural" TSEE peaks. For $X^{+}$and $X^{-}$planes of a crystal, the TSEE current density was found to differ from each other by a factor of $2-2.5$ after electron bombardment and by a factor of 5 after X-ray excitation. The TSEE from opposite surfaces of $Y$ - and $Z$-cuts were almost the same. The adsorption TSEE peak at $120^{\circ} \mathrm{C}$ had a maximum intensity for the (0001) surface.

\section{Energy spectrum.}

The energy distribution of exoelectrons in the TSEE 
Table I. The mean energies of exoelectrons-from the quartz crystal and glass surfaces

\begin{tabular}{ccc}
\hline surface & $\begin{array}{c}\text { Electron bonbardment } \\
1 \mathrm{keV}, 10 \mu \mathrm{A} \\
(\mathrm{eV})\end{array}$ & $\begin{array}{c}\text { X-raying } \\
\text { Dose } 1,2 \cdot 10^{4} \mathrm{R} \\
(\mathrm{eV})\end{array}$ \\
\hline$X^{+}(1 \overline{1} \overline{2} 0)$ & $0,99 \pm 0,05$ & $3,96 \pm 0,05$ \\
$X^{-}(\overline{1} \overline{1} 20)$ & $0,50 \pm 0,05$ & $0,86 \pm 0,05$ \\
$Y^{+}(10 \overline{1} 0)$ & $0,53 \pm 0,05$ & $0,46 \pm 0,05$ \\
$Y^{-}(\overline{1010)}$ & $0,47 \pm 0,05$ & $0,46 \pm 0,05$ \\
$Z$ (0001) & $0,90 \pm 0,05$ & $3,40 \pm 0,05$ \\
\hline Glass & $0,51 \pm 0,05$ & $0,53 \pm 0,05$
\end{tabular}

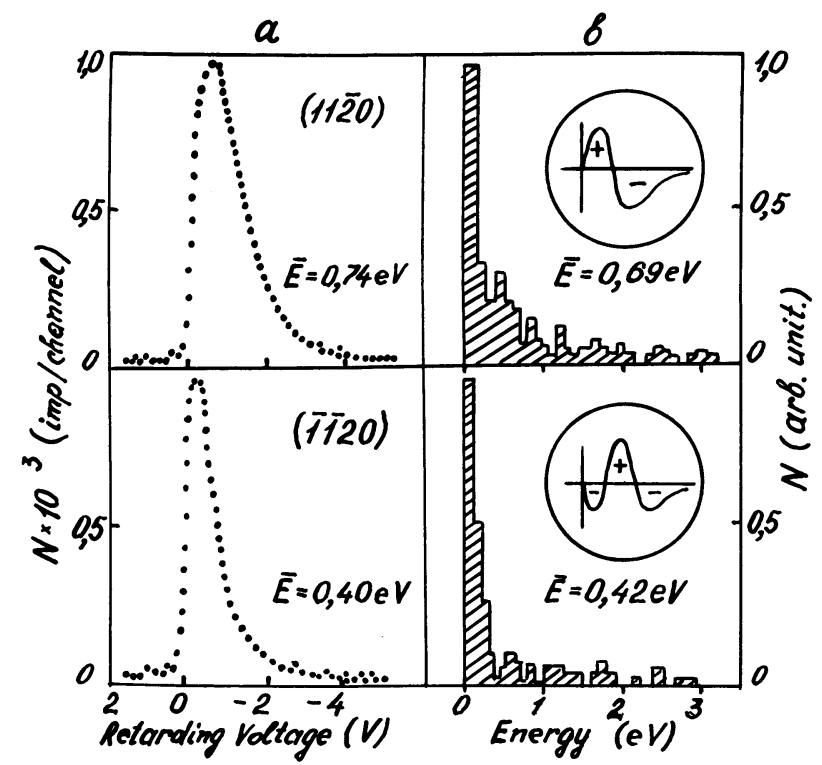

Fig. 2. Energy spectra of exoelectrons in the TSEE peak at $220^{\circ} \mathrm{C}$ for an $X$-cut after electron bombardment $(1,5 \mathrm{keV} ; 10 \mu \mathrm{A})$. a-Experimental spectra; $b$-theoretical spectra obtained by the MonteCarlo method for different space-charge distributions.

peak at $220^{\circ} \mathrm{C}$ also depended on the crystal orientation (Table I).

The greatest differences in electron mean energies were registered between the $(11 \overline{2} 0)$ and $(\overline{1} \overline{1} 20)$ planes. In this case, the increased energy values were characteristic for electrons emitted in the positive direction of the crystal $X$-axis (Fig. 2). The EDE parameters for the $Y$-cut surfaces differed slightly after electron bombardment, but coincide after $X$-raying the sample. The energy spectra for (0001) and (0001) surfaces of $Z$-cut were identical to each other for any type of excitation. The scatter in parameters for different quartz glass samples did not exceed the measurement error.

Figure 3 shows the change of the mean electron energy with the cutting angle successively relative to the crystal plane $(2 \overline{1} \overline{1} 0)$. A periodic variation of the mean energy with an interval of $120^{\circ}$ was observed. A similar periodic dependence for other crystal symmetry axes can be obtained in the same way. Figure 4 summarizes these results for samples excited by X-ray and electron bombardment.

In a three-dimensional presentation, the orientational dependence of the exoelectron energy has very complex forms which, as is evident from Fig. 4, have common symmetry elements with the crystal lattice. In the case of
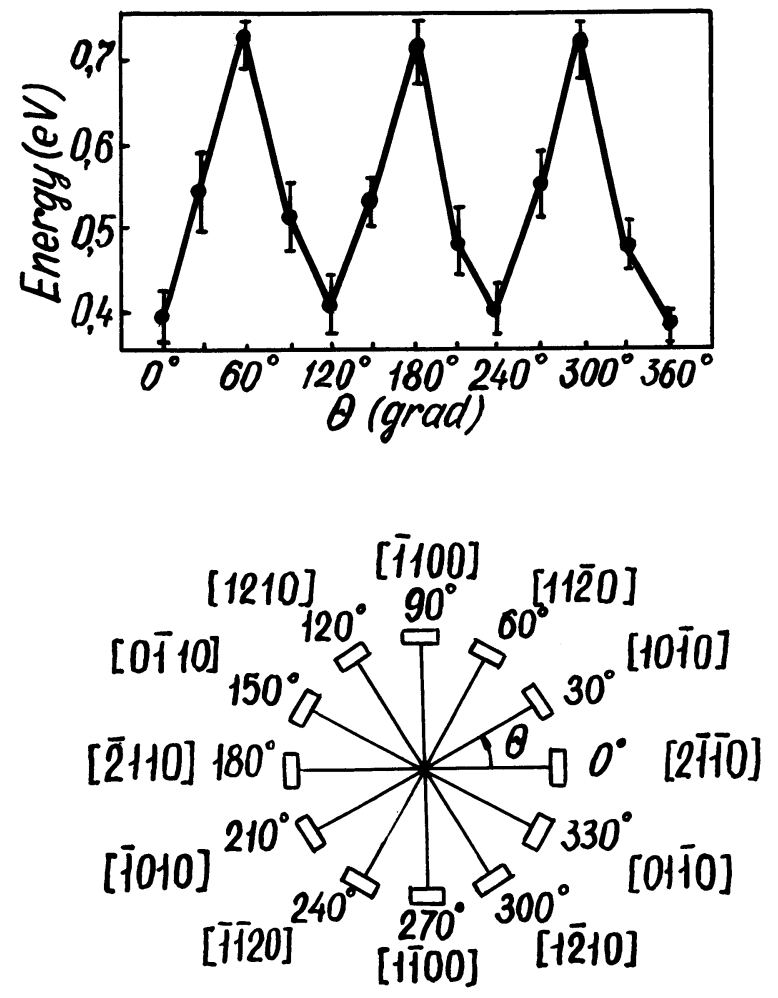

Fig. 3. The periodical change of the mean exoelectron energy in the TSEE peak at $220^{\circ} \mathrm{C}$ versus the cutting angle for the samples after electron bombardment $(1,5 \mathrm{keV} ; 10 \mu \mathrm{A})$.

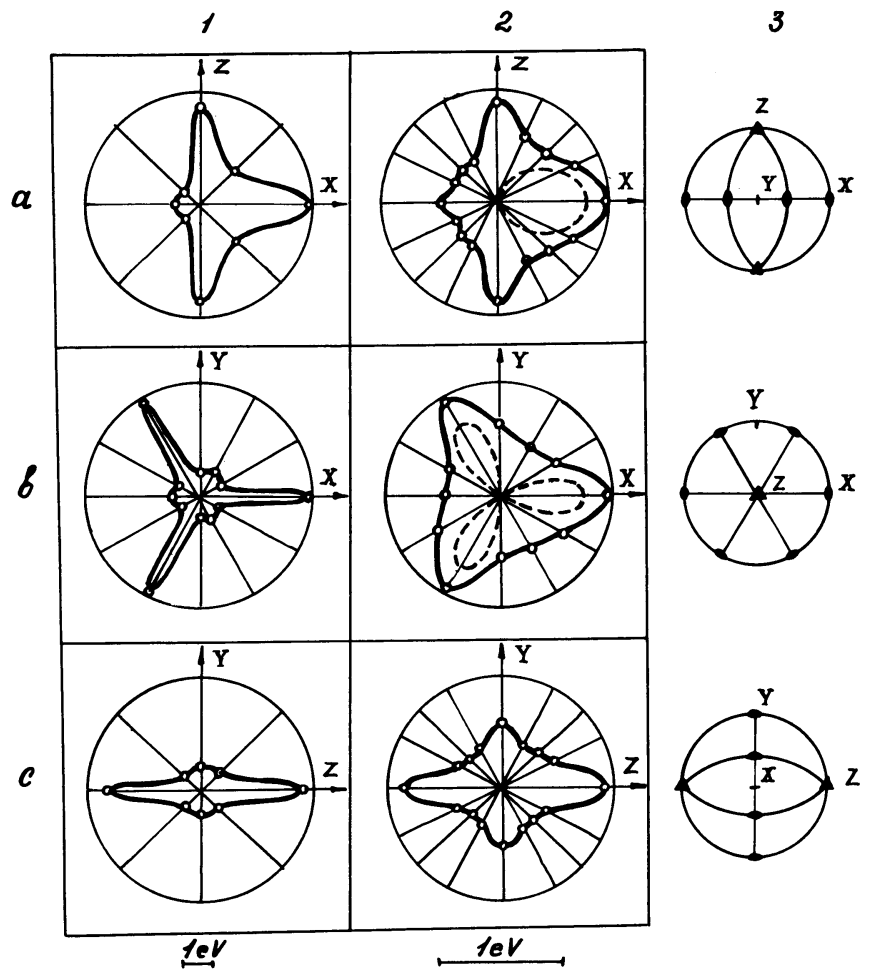

Fig. 4. Orientational dependencies of exoelectron energy: $a-(1010)$ section; $b-(0001)$ section; $c-(11 \overline{2} 0)$ section. $1-X$-ray excitation. 2-Electron bombardment. The symmetry of longitudinal piezoeffect is shown by dashed lines. 3-Stereograms of quartz symmetry elements. 
glass, such a dependence has a spherical shape. The established regularities were valid both for clean surfaces and for surfaces with adsorbates.

\section{§4. Discussion}

Symmetrical aspects.

A geometrical interpretation of the tensor properties is used for representation of anisotropy in crystal physics. ${ }^{9,10)}$ An investigation of the orientational dependence of the TSEE from quartz allowed us to suppose that the tensor approach could be applied to exoemission as well. The basis of such a conclusion was the correspondence between the orientational dependence of electron mean energies and the symmetrical elements of the point group of quartz. In other words, emission properties were closely associated with crystal macrosymmetry, i.e. they obeyed the well-known Neumann principle. ${ }^{10)}$ These important results allowed us to analyze the emission mechanism from quartz while taking into account the limitations caused by crystal symmetry. ${ }^{8}$

The nonequivalency of the energy states of different crystal planes can lead to an anisotropy of the exoemission properties. In principle, high-index crystal planes with a low reticular density and work function should possess an increased emission activity. However, it is known that the emission parameters of dielectrics depend on the electric field $\xi_{\text {ex }}$ due to the space charge formed during excitation. ${ }^{4)}$ In its turn, the polarization value of an excited sample depends on the dielectric constant $\varepsilon$.

In general, the dielectric constant as well as thermal expansion and conduction are represented by 2 nd-rank tensors which have a symmetry-of-revolution ellipsoid with an axis along the $\langle 0001\rangle$ direction in the quartz lattice. ${ }^{9)}$ Hence, the anisotropy of the physical properties mentioned above can not be the main reason of the observed orientational dependences of the TSEE. Since a quartz lattice has no symmetry center, the TSEE parameters are additionally affected by termally induced polarization. Such effects in TSEE are observed for piezoelectric crystals. ${ }^{5,6,8,11)}$ It follows from Fig. 4b that the symmetry of orientational dependence of the EDE parameters coincides with that of the longitudinal piezoeffect in quartz, which is represented by a 3rd-rank tensor. ${ }^{9)}$ Thus, on the basis of a symmetrical analysis, the observed anisotropy could be explained, first of all, by the origin of thermally induced piezoelectric polarization (secondary pyroeffect).

\section{Polarization effect.}

This effect, occurring during a linear heating of an excited crystal, produced a difference both in the internal electric field along the piezoactive directions (e.g. $\langle 11 \overline{2} 0\rangle$ and $\langle\overline{1} 120\rangle$ for $X$-cut) and in the potential barrier on the opposite sample surfaces.

Since for a free and "disconnected" crystal the electric induction $\boldsymbol{D}$ is zero and the polarization $\boldsymbol{P}$ is equal to the density of piezocharge $\sigma_{\mathrm{p}}$, then the equation is direct piezoelectric effect for $X$-cut is

$$
P_{\mathrm{x}}=e_{11} \cdot r_{11}-e_{11} \cdot r_{22}+e_{14} \cdot r_{23}
$$

where $e_{\mathrm{ij}}$ and $r_{\mathrm{ij}}$ are the components of the piezocoefficients and the deformation tensors, respectively. The value of sample deformations, which appear in the course of the TSEE process, was calculated from equation $r=\alpha \cdot \Delta T$, where $\alpha$ is the thermal expansion coefficient. The temperature range $\Delta T=\beta \cdot \Delta \tau$ is fixed by the experimental conditions (heating rate $\beta$, time $\Delta \tau$ which is taken by one order of magnitude less than the Maxwellian relaxation time). The piezofield intensity $\xi_{\mathrm{p}}$ in a crystal subsurface layer can be calculated from the formula for an infinite charged plane $\xi_{\mathrm{p}}=2 \pi \sigma_{\mathrm{p}} / \varepsilon \varepsilon_{0}$. After substituting all the values, we obtained $\xi_{\mathrm{p}}=0.6 \cdot 10^{4} \mathrm{~V} / \mathrm{cm}$ for $X$-cut.

The obtained $\xi_{\mathrm{p}}$ is quite sufficient to change the migration conditions and the departure of electrons released from bulk-emission centers. Depending on the crystal orientation, the field $\xi_{\mathrm{p}}$ will be accelerating ( $X^{+}$-cut), retarding ( $X^{-}$-cut) or equal to zero ( $Z$-cut). It is evident that the resulting field $\xi=\xi_{\text {ex }}+\xi_{\mathrm{p}}$ causes a TSEE processes with both a positive and a negative sign regarding the surface piezocharge. Thus, the TSEE process from a piezoactive surfaces takes place under conditions of the "plus-minus" and "minus-plus-minus" space-charge distributions. The results of computer calculations of the exoelectron energy spectra for these space-charge distributions agree with experiment (Fig. 2b).

\section{The bending of energy bands.}

In the course of heating, the piezocharge generation causes a band bending near the surface. This can be calculated from the experimental TSEE curves. For the TSEE thermal activation process, the exoemission current can be expressed by

$$
\begin{aligned}
I= & B \cdot p_{0} \cdot C \cdot \exp \left(-\frac{E+\chi \pm \varphi}{k T}\right) \\
& \cdot \exp \left\{-\frac{p_{0}}{\beta} \int_{0}^{T} \exp \left(-\frac{E}{k T}\right) \mathrm{d} T\right\},
\end{aligned}
$$

where $B$ is the constant, $p_{0}$ is the frequency factor, $C$ is the concentration of emission centers, $E$ is the energy depth of the center, $\chi$ is the electron affinity, $\varphi$ is the bending of the bands.

For the $X^{-}$and $X^{+}$surfaces the $I^{+} / I^{-}$gives the difference in the band bending due to the piezoelectric effect for opposite planes; $\Delta \varphi=0.03 \sim 0.06 \mathrm{eV}$ and $\Delta \varphi=0.07 \sim 0.10 \mathrm{eV}$ after electron and X-ray excitation, respectively. The observed dependence of the band bending and EDE parameters on the type of excitation results from a difference in the ionizing ability of electrons and X-rays. The relatively small values of $\Delta \varphi$ together with the well-defined anisotropy of the EDE parameters, indicate the influence of surface conductivity on the TSEE intensity. This allows us to suggest that the maximum density of the piezopolarization charge is situated in the depth of the subsurface emission-active layer.

\section{§5. Conclusions}

Investigations with quartz allowed us to demonstrate the efficiency of the approach taking into account the crystallographic axis dependence for understanding the nature of exoemission effect in anisotropic dielectrics. 
The discovered correlation between the point symmetry group of a crystal and the orientational dependence of the TSEE parameters evidently has a general meaning and, thus, forms the basis for the prediction of some emission peculiarities in single crystals.

Application of the mean exoelectron energy to a measure of the anisotropy of exoemission permitted us to determine the role of symmetry effects for the TSEE mechanism of quartz. From the obtained results, it follows that the emission mechanism from the crystal piezoactive planes is determined by a change in the charge distribution in the emission-active crystal layer which is caused by the piezocharge and an additional polarization of piezoelectric origin. Moreover, the data indicate that the anisotropy of exoemission parameters was due to of nonequivalent conditions, both regarding the electrons departure from the emitter surface and the electron movement in the subsurface layer.

\section{Acknowledgement}

We would like to thank V. G. Isakov for his assistance regarding computor calculations.

\section{References}

1) K. Tsumori, S. Okabe and T. Seiyama: Ann. Report Rad. Center Osaka Prefect. 18 (1977) p. 29.

2) A. F. Zatsepin and V. I. Ushkova: Exoelectron Emission and Applications (Sverdlovsk, 1979) p. 24.

3) T. Kubozoe, Y. Waranabe, Y. Nakamura, S. Okabe and M. Aoki: Rad. Protect. Dosimetry $43 / 4$ (1983) 257.

4) V. Kortov, V. Isakov, H. Glaefeke and H. -I. Fitting: phys. stat. sol. (a) 73 (1982) K 275.

5) V. I. Ushkova, V. A. Kalentyev and A. F. Zatsepin: RadiationStimulated Processes in Solids (Sverdlovsk, 1983) p. 18.

6) A. F. Zatsepin, V. A. Kalentyev and V. S. Kortov: Pisma v Zhurn. Tekhn. Fiz. 10 (1984) 102.

7) A. B. Gerasimov, M. M. Merkin and A. A. Tsertsvadze: phys. stat. sol. (b) 128 (1985) 693.

8) A. F. Zatsepin, V. S. Kortov and V. A. Kalentyev: Proc. 19th Conference on Emission Electronics (Fan, Tashkent, 1984) p. 190.

9) I. S. Zheludev: Symmetry and its Applications (Energoatomizdat, Moscow, 1983) p. 141.

10) J. F. Nye: Physical Properties of Crystals (Mir, Moscow, 1967) p. 157.

11) P. A. Vaivod, A. F. Zatsepin, V. A. Kalentyev, V.S. Kortov and K. K. Shvarts: Izv. Akad. Nauk Latv. SSR, ser. fiz. 4 (1984) 40. 